\title{
Germanica
}

\section{Eine fremde Welt: H. G. Adler, Eine Reise}

Un monde étrange : H.G. Adler, Eine Reise

\section{Ruth Vogel-Klein}

\section{OpenEdition}

Journals

Édition électronique

URL : http://journals.openedition.org/germanica/508

DOI : $10.4000 /$ germanica.508

ISSN : 2107-0784

Éditeur

Université de Lille

\section{Édition imprimée}

Date de publication : 1 juin 2008

Pagination : 13-28

ISBN : 978-2-913857-21-6

ISSN : 0984-2632

Référence électronique

Ruth Vogel-Klein, «Eine fremde Welt: H. G. Adler, Eine Reise », Germanica [Online], 42 | 2008, Online erschienen am: 01 Juni 2010, abgerufen am 07 Oktober 2020. URL : http://journals.openedition.org/ germanica/508; DOI : https://doi.org/10.4000/germanica.508

Ce document a été généré automatiquement le 7 octobre 2020.

(c) Tous droits réservés 


\title{
Eine fremde Welt: H. G. Adler, Eine Reise
}

\author{
Un monde étrange : H.G. Adler, Eine Reise
}

Ruth Vogel-Klein

1 H.G. Adler, geboren 1910 als deutschsprachiger Jude in Prag, gestorben 1988 in London, wohin er 1947 ausgewandert war, kann zu den großen europäischen Intellektuellen des 20. Jahrhunderts gezählt werden. Von seinem vielfältigen Werk sind vor allem die Bücher über die nationalsozialistische Judenverfolgung bekannt. Einst selber ein Verfolgter, hat Adler auf verschiedenste Weise Zeugenschaft abgelegt. Seine wissenschaftlichen Bücher Theresienstadt 1941-1945. Antlitz einer Zwangsgemeinschaft ${ }^{1}$, Theresienstädter Dokumente ${ }^{2}$ und Der verwaltete Mensch. Studien zur Deportation der Juden aus Deutschland ${ }^{3}$ sind grundlegende Werke zur nationalsozialistischen Judenverfolgung. Darüber hinaus ist Adler Autor einer Reihe von literarischen und essayistischen Werken. All diese Bücher stellten einen wichtigen Beitrag zu der bundesdeutschen Auseinandersetzung mit dem Nationalsozialismus und der Shoah dar, die allgemein «Vergangenheitsbewältigung» genannt wird. Dieser Begriff als solcher ist problematisch, denn er scheint die Überzeugung widerzuspiegeln, dass man diese Vergangenheit hinter sich lassen könne, denn Bewältigen impliziert prinzipiell das Überwinden und ad acta legen ${ }^{4}$. Tatsache ist jedoch, dass 2008, zu einer Zeit der intensiven Auseinandersetzung mit der NS-Zeit, die kollektive und individuelle Konfrontation der Deutschen mit der nationalsozialistischen Vergangenheit meist unter dieser Bezeichnung zusammengefasst wird. Peter Reichel unterscheidet in seinem Buch Vergangenheitsbewältigung in Deutschland. Die Auseinandersetzung mit der NSDiktatur von 1945 bis heute vier «Handlungsfelder der Vergangenheitsbewältigung» : die politisch-justitielle Auseinandersetzung mit den Folgen der Hitler-Diktatur, die Geschichte der öffentlichen Erinnerungs - und Memorialkultur, die wissenschaftliche Auseinandersetzung mit der Geschichte des Nationalsozialismus und die «Geschichte der ästhetischen Kultur, also die subjektive Vergegenwärtigung der NS-Vergangenheit in den künstlerischen Medien» ${ }^{5}$. 
2 In den beiden letzteren kollektiven Handlungsfeldern hat H.G. Adler eine wichtige Rolle gespielt, als Wissenschaftler einerseits und als Schriftsteller und Dichter andererseits. Bei Adler geht es jedoch auch um Vergangenheitsbewältigung als individuellen psychischen Akt im Sinne eines «Durcharbeitens» ${ }^{6}$. Adler hat die Bewältigung der Deportation und des Vernichtungsgeschehens durch Schreiben mehrfach thematisiert. In seinem Text «Warum habe ich mein Buch ,Theresienstadt 1941-1945` geschrieben?» heißt es:

Ich fühlte, daß ich nicht mehr fortdauern könnte, daß der Schmerz über das Geschehene eine abgründige Verzweiflung in gähnender Leere in mir zurücklassen müßte, wenn ich nicht auf diese Weise mich bestrebte, das Ungeheuerliche seelisch und geistig zu bewältigen: es blieb mir gar nichts anderes übrig, als meine Forschung zu beginnen?

Auch Eine Reise kann trotz seiner Unterschiede zu Theresienstadt 1941-1945 an dieser Stelle angeführt werden. Kennt man die Biografie Adlers, so finden sich in Eine Reise verfremdet, transponiert und erweitert, Elemente seiner Erfahrung der Shoah wieder ${ }^{8}$, die er in dem Roman durch Schreiben zu bewältigen suchte. Auch dieser Text ist, wie das Theresienstadt-Buch, eine Würdigung der Toten und eine Anklage der Mörder. Marcel Atze schreibt:

Adler wollte der Judenvernichtung ein Narrativ geben, mithin Gedächtnisarbeit leisten. Ihm war keineswegs entgangen, dass dem Genozid wohl der Memorizid gefolgt wäre, nicht nur um das jüdische Gedächtnis zu vernichten, sondern auch um die Erinnerung an die Juden zu manipulieren [.... ${ }^{9}$

\section{Biographisches}

4 Geboren wurde Hans Günther Adler noch als Österreicher des Habsburgerreichs in einer deutschsprachigen jüdischen Familie in Prag. Obwohl nach 1918 die deutsche Kultur in der unabhängigen Tschechoslowakei immer mehr zurücktrat, bestanden noch deutsche Schulen und die deutschsprachige Universität in Prag, an der Adler Musikund Kunstwissenschaft, Philosophie, Psychologie und Germanistik studierte und 1935 promovierte. Die Wege zu einer Hochschulkarriere verschlossen sich ihm 1938 mit der Besetzung der Tschechoslowakei durch die Nationalsozialisten. Nachdem Auswanderungspläne gescheitert waren, wurde er 1941 zuerst als Zwangsarbeiter beim Eisenbahnbau eingesetzt, dann 1942 mit seiner Frau, der Ärztin Gertrud Klepetar, und deren Eltern deportiert. Auch seine Eltern wurden verschleppt und umgebracht; von seiner Familie und derjenigen seiner Frau kamen achtzehn Menschen nicht aus der Deportation zurück. Er allein überlebte die Transporte in verschiedene Lager, zunächst nach Theresienstadt, dann nach Auschwitz, Niederorschel und Langenstein, bis zu der Befreiung durch die Amerikaner. Schon im KZ fasste Adler den Entschluss, über diese Zwangsgemeinschaft zu schreiben. Den Namen Hans Günther legte er ab, da der Vertreter Eichmanns in Prag diesen Namen getragen hatte, fortan schrieb er sich H. G.

1945 ging Adler nach Prag zurück, in der vergeblichen Hoffnung, dort seine Frau noch wiederzufinden. Er arbeitete an dem Neuaufbau des Prager Jüdischen Museums mit und betreute auch Waisenkinder aus den Lagern ${ }^{10} .1947$ verließ Adler endgültig Prag. Aufgrund seiner deutschen Muttersprache hatte er inzwischen die tschechische Staatsbürgerschaft verloren, darüber hinaus befand er sich im Gegensatz zu dem neuen Regime. Von nun an lebte er in London als freier Schriftsteller und Wissenschaftler, oft unter materiell schwierigen Bedingungen ${ }^{11}$. Die deutsche Verlagswelt wies seine Werke 
lange $a b$, und Adler musste viele Widerstände überwinden, damit seine Bücher gedruckt werden konnten. Das Londoner Exil erschwerte die Kontakte, aber vor allem war es sein Thema der Judenverfolgung, fern vom main stream der damaligen Publikationen, das ihm die Türen verschloss. Joachim Campe, der das mangelnde Echo auf Adlers Werk in den fünfziger und sechziger Jahren zu erklären versucht, schreibt über diese Zeit, dass die Bundesrepublik nicht an das «Dritte Reich» erinnert werden wollte, «man betrachtete es als ein unbegreifliches Verhängnis der Geschichte, an dem eigentlich niemand schuld gewesen war» ${ }^{12}$. Die Kontinuität der herrschenden westdeutschen Eliten in Wirtschaft, Politik und Kultur spielte bei der damaligen Ausblendung der NS-Zeit eine wesentliche Rolle, und Alexander und Margarethe Mitscherlich sind in ihrer berühmten Studie Die Unfähigkeit $z u$ trauern den psychosozialen Hintergründen dieses Wegschauens nachgegangen ${ }^{13}$.

\section{Eine Reise: Grundlinien}

6 Als Adler 1951 den Roman Eine Reise abschloss ${ }^{14}$, hatte er schon drei Werke niedergeschrieben, die damals noch unveröffentlicht waren. Für die umfangreiche wissenschaftliche Studie Theresienstadt 1941-1945, Antlitz einer Zwangsgemeinschaft aus dem Jahr 1948 hatte er schon im Lager unter schwierigsten Bedingungen Material gesammelt, das er später in Prag noch vervollständigte. Der Roman Panorama, ebenfalls 1948 abgeschlossen ${ }^{15}$, rollt in 10 „Bildern“ die Lebensgeschichte des Artur Landau auf, die stark autobiographische Züge trägt. Während diese beiden Bücher später noch Verleger fanden, ist der Roman Die Ansiedlerin (1949) noch heute unveröffentlicht. Wenn auch z. T. gewisse thematische und inhaltliche Verbindungen dieser Werke $\mathrm{zu}$ Eine Reise bestehen, so ist dieses sperrige Buch doch schwer zuzuordnen und siedelt sich auf einer anderen Darstellungsebene an. (Eigentlich sollte das Buch Die Reise heißen, aber der Titel war schon vergeben.) Wegen seiner lyrisch-fiktionalen Schreibstrategien schlug der Autor als Untertitel zu Eine Reise erst «Sage», dann «Ballade» vor, was sein Verleger jedoch abwies. Sie einigten sich dann auf den Untertitel «Erzählung», was aber Heimito von Doderer zu Recht anfocht:

Der Autor nennt dieses Buch «Erzählung». Es ist ein Roman. Keineswegs des

Umfangs wegen, sondern durchaus in Anbetracht des universalen Zugriffs. ${ }^{16}$

Eine Reise ist ein vielstimmiger Roman über die Deportation, mit einer Haupthandlung, der Geschichte der Arztfamilie Lustig, und einer ganzen Anzahl von Nebenhandlungen. Dr. Leopold Lustig, seine Frau Karoline, Tochter Zerline und die Verwandte Ida Schmerzenreich kommen im Lager um, nur der Sohn Paul überlebt. Die Zeitstruktur ist komplex, häufige Analepsen und Prolepsen durchbrechen den Fluss der Erzählgegenwart. Um dem Leser erste Anhaltspunkte zu liefern, könnte man den Text grob gesehen in drei Teile gliedern: 1. die Abreise oder der Abtransport der Familie Lustig aus der Stadt Stupart 2. die Zeit in den Lagern und 3. das Überleben Pauls, der in eine neue Zukunft aufbricht. Diese Zusammenfassung nimmt eine extreme Vereinfachung vor, denn die ganze polyphon aufgefächerte Handlung gründet sich auf das Prinzip des Impliziten, Vorgewussten und oft Doppeldeutigen. Zu den wesentlichen Charakteristika des Romans gehören auch ein verfremdender Sprachgebrauch und eine symbolhafte Namensgebung. 


\section{Namen} anmutenden Form zunächst antiphrastisch. Aber darüber hinaus klingt in dem Namen auch deutlich der Friedhof an, auf dem die Toten ruhen ${ }^{19}$. Beide Bedeutungen bestehen nebeneinander, Adlers Schreibstrategien sind nie einschlichtig. Bei dem Familiennamen Lustig kann zunächst ebenfalls die Umkehrung des Sinns festgehalten werden. Vier Romanfiguren, die Eltern Karoline und Leopold, sowie ihre Kinder Paul und Zerline, die alle brutal aus ihrer Heimat vertrieben werden und dem schrecklichen Schicksal der Deportation ausgeliefert sind, tragen diesen Nachnamen. Die sehr häufige Nennung des Namens Lustig schafft eine immer erneuerte Kollision zwischen der Welt des Todes, der Erniedrigung und Entrechtung einerseits und einer irrealen heiteren, komödienhaften Welt andererseits. Das Verschwinden dieses Namens auf dem Arztschild auf der Hauswand, das dem Verschwinden der Personen vorausgeht, wird in dem Buch graphisch und sinnmäßig hervorgehoben. Es geht in den folgenden Zeilen um den Augenblick, als die Familie Lustig dem Deportationsbefehl folgt und die Treppe ihres Hauses zum letzten Mal hinuntergeht.

Jetzt aber war man an einer unsicheren Stelle. Es war keine Stelle mehr, und vielleicht fehlte auch jeder Zusammenhang mit einer Adresse, seitdem man das Schild

Dr. Leopold Lustig

Praktischer Arzt

entfernt hatte; jetzt aber sollte auch nichts mehr dasein, was noch etwas gewesen wäre. Also war gar nichts mehr. Damit wollte sich die Erinnerung nicht zufrieden geben, die sich stets um Einteilungen bemüht ${ }^{20}$.

Die Vernichtung des Namens lässt alle Anhaltspunkte und Verortungen zusammenbrechen.

«Lustig» erscheint den Romanfiguren darüber hinaus die Haltung der vollkommen realitätsfernen Figur dieses Arztes Leopold Lustig, über den sich die andern Häftlinge lustig machen, als er in dem unmenschlichen Sammellager gegen die Organisation der Reise lauthals protestiert und eine Kampferspritze für eine gestörte Frau verlangt ${ }^{21}$. Der Name Lustig besitzt noch eine andere Dimension, indem er mit dem Namen der Karoline Lustig, die eine geborene Schmerzenreich ist, die Antinomie der beiden Figuren Karoline und Leopold hervorhebt. Zudem wird die Deplatziertheit des Namens Lustig durch die Konfrontation mit Schmerzenreich noch unterstrichen. In dem Namen Schmerzenreich findet einerseits eine Metaphorisierung des Lagerdaseins als Reich des Schmerzens stat $t^{22}$, wie Jeremy Adler schreibt, andererseits wird das ganze traurige Schicksal der Karoline versinnbildlicht - ihre unglückliche Ehe mit dem Arzt Leopold, ihre Verschleppung und der Tod im Lager. Das Schicksal der Schwester Ida 
Schmerzenreich fügt noch die Konnotation des körperlichen Schmerzes hinzu, denn Ida, übrigens eine verwitwete Frau Schwarz, hat sehr schweren Rheumatismus, der ihr Schmerzen bereitet. Durch den Namen Schmerzenreich wird auch eine christliche Intertextualität eingesetzt, denn die Schmerzenreiche ist bekanntermaßen die Gottesmutter Maria der katholischen Tradition, wie zahlreiche Gebete belegen. In der Literatur ist es Goethe, der in dem Faust I Gretchen mit folgenden Worten beten lässt:

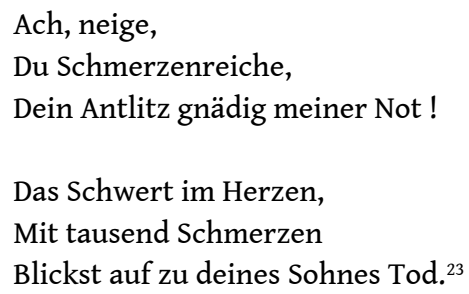

Das Leid der jüdischen Frauen Karoline und der Ida Schmerzenreich, die als Mütter nicht wie Maria ihren Sohn verlieren, sondern selber zugrundegehen, während der Sohn überlebt, kehrt den christlichen Begriff der mater dolorosa um. Die überlebende Maria hat in diesem Text ihren Platz verloren, der Sohn wird kein Erlöser, und der Schmerz ist derjenige der verfolgten und ermordeten jüdischen Frauen. Dies mag als eines der vielen Beispiele im Roman für eine kritische Konfrontation mit der christlichen Religion und der Kirche gelesen werden.

Ein bedeutsamer Intertext liegt ebenfalls dem Vornamen Zerline zugrunde. Brochs Erzählung der Magd Zerline, die 1950 erschienen war $^{24}$, scheint nicht zu passen, plausibler erscheint, dass der Musikwissenschaftler Adler an die Bäuerin Zerline aus dem Don Giovanni gedacht hatte ${ }^{25}$. Adlers Zerline spielt Laute, und auf eben diesem Instrument begleitet sich der Don Giovanni, als er der Braut Zerline ein Ständchen bringt. Vergleichbar dem Namen Lustig, gewinnt der Name Zerline bei Adler seine Bedeutung vor allem durch seine Deplatziertheit. Das Ende aller Ständchen, aller Mozartarien und der schelmisch-heiteren Zerlinefiguren wird in einer der Anfangsszenen von Eine Reise durch die Beschlagnahmung aller Musikinstrumente bezeichnet. Die Bitterkeit dieser Maßnahme kristallisiert sich in der Weigerung Pauls, das Instrument heil abzuliefern und seinem Widerstandsakt der Zerstörung der abzuliefernden Laute.

Paul riß mit fünf von den sechs Saiten der Laute die Seele aus und schnitt mit einem Messer ihr empfindliche Wunden in den Leib. Zerline klagte über den Mord, die Laute sei doch kein Abfall. Jetzt aber war sie es, niemand wird sich an ihren Tönen mehr freuen. Zuletzt zog Paul noch einen Wirbel heraus und warf ihn ins Feuer ${ }^{26}$.

Man könnte in dieser Szene eine Vorausdeutung auf den Tod Zerlines sehen: das Lied ist aus, wie es später im Roman mehrmals heißt. Die Laute, die man als eine Metonymie für das junge Mädchen sehen kann, reiht sich in ihrer Degradierung zum Abfall in das Adlersche Leitmotiv der zum Abfall abgestempelten Menschen ein.

Nach den komplex miteinander verschränkten antiphrastischen, symbolischen und intertextuellen Namensgebungen kann der Name Zerline auch als Beispiel für eine bedeutsame Assonanz genannt werden. Jeremy Adler weist darauf hin, dass H. G. Adler seine erste Frau Gertrud Klepetar Geraldine nannte. «Hier erhält sie den verklärenden Namen Zerline ${ }^{27}$.» Geraldine/ Zerline : durch diese Assonanz setzt der Autor einen literarischen Gedenkstein für die Verstorbene. Andersgeartete Assonanzen erfindet Adler mit dem Ortsnamen Leitenberg, der Stadt in der Nähe Ruhenthals/ Theresienstadts. Der Name erinnert an das Wort «Leichenberg» oder auch 
«Leidenberg» und bildet im Übrigen einen der wenigen approximativen Hinweise auf die geographische Realität: die Ortschaft bei Theresienstadt hieß auf Deutsch Leitmeritz.

\section{Eine fremde Welt} schrecklichen Ort Ruhenthal, in der Perspektive des heterodiegetischen Erzählers und der verschleppten Menschen. Durch den inadäquaten Begriff der Reise, über den der Leser immer wieder stolpert, hebt Adler stets erneut die Unglaublichkeit des Deportationsgeschehens hervor. Der alltägliche Begriff bezeichnet die tiefe Kluft zwischen dem Altbekannten einerseits, der Grausamkeit der Verschleppung und Vernichtung andererseits. Über diese grundlegende Verfremdungsfunktion hinaus, die wie bei Brecht eine Bewusstwerdung auslösen soll, kommen dem Titelwort «Reise» noch andere Funktionen zu, je nach der häufig wechselnden Erzählperspektive.

Im Munde der Täter und Mitwisser entspricht die Bezeichung «Reise» einer bewussten Verschleierung. In dem folgenden Passus erscheint eine Stimme, die den Schergen der 
Deportations-Sammelstelle zugeordnet werden kann. Die beängstigende Situation des Aufbruchs zur «Reise» wird folgendermaßen kommentiert:

$\mathrm{Ja}$, alles wird euch nachgeschickt, was euer Herz begehrt. Nur fahren sollt ihr schon endlich! Vergessen habt ihr was? Das wird schon nicht so wichtig sein. Schreibt Briefe von dort, wir werden alles bestellen. Wir sind verläßlich. Seht ihr es nicht unsern Gesichtern an? Unsere Augen müssen euch unsere Treue verrraten! Es könnte euch etwas geschehen? Wer sagt euch das? Alles dummes Geschwätz. Noch keinem wurde ein Härchen gekrümmt. Jetzt fahrt ihr in die Sicherheit, in eure Heimat, wie ihr es immer gewünscht habt. [...] Das Verbotene liegt nun endgültig hinter euch, da euch die ewige Freiheit winkt. Dort dürft ihr. Wir wünschen es uns selber, euer Schicksal zu teilen, aber leider ist uns das versagt. Uns ruft die Pflicht, denn wir müssen weiter für euer Wohl sorgen, und dann sind noch eure Brüder zu versorgen, die auch schon auf die Reise warten ${ }^{29}$.

Eine Isotopie der Geborgenheit und des Unverdächtigen wird an der Oberfläche entwickelt $^{30}$, jedoch auf diverse Weise unterminiert. Dem aufmerksamen Leser erschließt sich eine zweite Bedeutungsebene, durch die das Unheimliche unterschwellig in die Rede einsickert. Einerseits bestehen semantische Inkohärenzen innerhalb des Abschnitts. Die vielen Beteuerungen, dass den Reisenden nichts geschehen wird, verkehren sich in ihr Gegenteil und schaffen eine Verunsicherung. Und wie ist es zu verstehen, dass die Familie, die aus ihrer Heimat Stupart abreisen muss, in die «Heimat» fährt? Und kann man nicht das Versprechen der ewigen Freiheit so verstehen, dass am Ende der «Reise» die Ewigkeit steht, der Tod, der zynisch als Heimat bezeichnet wird? Andererseits wird der ganze Passus über die vertrauenerweckende Reise von der bitteren Ironie getragen, die das ganze Buch charakterisiert. Für die Definition der Ironie soll Philippe Lejeune angeführt werden.

Eine ironische Aussage ist eine Aussage, in der man etwas anderes sagt, als man denkt und gleichzeitig etwas anderes zu verstehen gibt, als man sagt. Ihre Funktion ist, den Diskurs des andern zu subvertieren: man entlehnt wörtlich die Aussagen des Gegners, schafft jedoch einen Bruch in Kontext, Stil oder Ton, der sie virtuell unsinnig, abscheulich oder lächerlich erscheinen lässt und der implizit zum Ausdruck bringt, dass das Aussagesubjekt einen anderen Standpunkt vertritt ${ }^{31}$.

Zunächst entsteht die Ironie in dem obigen Zitat Adlers durch den Widerspruch zum Kontext. Hier ist der unmittelbare Kontext die unmenschliche Unterbringung von Hunderten von Menschen im Technischen Museum ${ }^{32}$, die Deportation von Kranken, die Erniedrigung bei dem Zählappell ${ }^{33}$. Über diese Diskrepanz zwischen Passus und Kontext hinaus greift Adler, um den Betrug der verschleppten Personen umso schärfer hervortreten $\mathrm{zu}$ lassen, $\mathrm{zu}$ dem ironischen Kunstgriff, der darin besteht, einen bestehenden Diskurs zu übertreiben, ihm scheinbar beizupflichten, seine abweichende Meinung jedoch zu markieren. Diese Markierung erfolgt sowohl durch die allzu treue und verlässliche Selbstdarstellung der Sprecher, als auch durch den anbiedernden Ton und die übertriebenen Hilfsangebote. Die schablonenhafte Sprache, wie z. B. «was euer Herz begehrt» oder «noch keinem wurde ein Härchen gekrümmt», «uns ruft die Pflicht», die von dem sehr differenzierten sprachlichen Kontext abweicht, ist ein Indikator von Lüge und Verstellung und schafft die ironische Differenz.

Aus der Perspektive der Opfer bezeichnet der Begriff «Reise» eine (von den Tätern vorgespiegelte) Hoffnung und Illusion, an der sich manche festhalten, so die Figur des alten Arztes Dr. Leopold Lustig. Vor der «Abreise» protestiert er (in dem Kontext der Schreckensherrschaft im Sammellager) gegen die Misshandlung einer kranken Frau: 
Den Fall muss man der Ärztekammer anzeigen! So geht man nicht mit Menschen um. Wenn ich gewusst hätte, dass hier so eine Ungerechtigkeit herrscht, so wäre ich zu Haus geblieben und hätte meiner Familie nicht erlaubt, sich an dieser Reise zu beteiligen. Die Vorbereitung ist miserabel ${ }^{34}$.

Die Ironie besteht hier nicht in der Unauthentizität der Sprache, sondern allein in dem Widerspruch $\mathrm{zu}$ dem Kontext, in der Gegenüberstellung eines vernünftigen und humanen Diskurses, der zutreffenderweise die Ungerechtigkeit benennt, mit der Situation der absoluten Rechtlosigkeit.

Die «Reise» ist auch eine Chiffre für Existentielles, sie bezeichnet, nach alter literarischer Tradition, den Weg in den $\operatorname{Tod}^{35}$ und, am Schluss des Romans, auch die Rückkehr des Paul Lustig in das Leben:

Paul beschließt, übermorgen Unkenburg zu verlassen, vielleicht schon morgen. Die paar Sachen, die für seine Reise ihm nützen können, will er morgen früh packen. [...] Die Reise lockt, sie ist gewiss. [...] Von der kleinen Wanderung heute ist er müde, doch er ist fröhlich, weil er reisen wird, wie er es will ${ }^{36}$.

Durch diese völlig neu besetzte Bedeutung des Begriffs auf den letzten Seiten des Buches wird dessen negative Bedeutung verwandelt und mit Hoffnung gefüllt. Die Reise als Deportation wird von Paul Lustig überwunden und erscheint so als eine Etappe eines Überlebensweges, der in die Zukunft führt.

\section{Fremdlinge und Verbotene}

Adlers fremde Welt, die wie ein Science-Fiction-Roman in eine räumliche und zeitliche Ferne entrückt ist, kennt keine historischen Benennungen wie SS, SA, Nationalsozialisten, Deportation, Juden, Konzentrationslager ${ }^{37}$. In den erdachten Bezeichnungen für Täter, Opfer und die gleichgültigen Mitwisser in dieser unbarmherzigen Welt drückt der Autor jeweils seine Weigerung aus, sich der bestehenden Diskurse zu bedienen.

Seine Begrifflichkeit bei der Bezeichnung der Menschenkategorien ist semantisch komplex.

In einer Umkehrung des antisemitischen Diskurses, der den Juden ihre Heimat bestritt und sie als «heimatlose Gesellen» verhöhnte, werden die Verfolger, welche die Familie Lustig abholen, als «Fremdlinge» bezeichnet. Dies hebt nicht nur das Einbrechen des Fremden in eine gewöhnliche Alltagswelt hervor; «Fremdlinge» sind sie tatsächlich in einer Welt des Rechts und der Menschlichkeit: sie begeben sich ja in Wohnungen, in denen sie fremd sind ${ }^{38}$, verletzen rechtswidrig zuerst die Privatsphäre einer Familie ${ }^{39}$, um sie dann zu verschleppen. In diesen Seiten der behördlichen Verfolgung und Entrechtung klingt durchaus Kafkas Process an. (Adler war schon in Prag ein begeisterter Kafka-Leser gewesen.) In der Sammelstelle für die Deportierten in der Stadt Stupart heißt der oberste Befehlshaber hingegen in einer scheinbaren Übernahme der herrschenden Ideologie «Held». Seine Handlungen, Redeform und inhalt sind jedoch unmenschlich, brutal und vulgär ${ }^{40}$, was den Begriff als ironische Antiphrase entlarvt ${ }^{41}$. Bei den Bezeichnungen für die Juden setzt der Autor wiederholt Begriffe ein, die scheinbar der Ideologie der Unterdrücker entstammen, doch auf verschiedene Weise überspitzt oder verfremdet sind. Zunächst sind die Juden die «Verbotenen». Adler fasst in dieser Formel die Absurdität der rechtlosen Existenz der Juden zusammen und antizipiert so am Anfang des Buches schon die kommenden 
Vernichtungsstrategien. Später im Lager sind sie die «Sklaven», ein scheinbar herrschaftskonformer Begriff, der sofort wieder problematisiert wird:

Die Sklaven genießen die Rechte ihrer Herren und sind noch in ihrer Ohnmacht großartiger daran als die Freien in ihrer Beschränkung. "Schaufeln und Spitzhacken holen!» Sie dürfen es, wenn sie es auch müssen. Die Sklaven sind frei. Sie können hören und gehorchen. Sie trinken Befehle und dürfen sie befolgen. ${ }^{42}$

Adler schafft, abgesehen von dem Kontext, in dem die unwürdigen Lebensbedingungen der Zwangsarbeiter doch erscheinen, subtile Widersprüche innerhalb des Passus'. Um die oxymorische Behauptung "die Sklaven sind frei» herum wird eine Argumentation entfaltet, die sich auf eine paradoxe Verwendung der Modalverben gründet. Die Logik des Passus wird so nachhaltig durchlöchert. Gleichzeitig mit dem Täterdiskurs entwirft Adler einen gegenläufigen Diskurs, der die «Ohnmacht» und die Zwangssituation der Sklaven («wenn sie auch müssen») fast unmerklich einfließen lässt.

Ein anderes Beispiel, bei dem ein bestehender Diskurs («wir haben nichts gewusst») ironisch auf die Spitze getrieben wird, ist die Bezeichnung des Zugs der erschöpften und zerlumpten Sklavenarbeiter durch die Stadt Leitenberg als «Geister», eine unheimliche Schar, die niemand gesehen haben will ${ }^{43}$. Gleichzeitig wird dieser Begriff so nachdrücklich über Seiten hinweg verwendet, dass tatsächlich eine befremdliche und unheimliche Geisterwelt vor den Augen der Leser ersteht. Wie Adler hier die ironische Bezeichnung «Geister» aus der Situation der KZ-Häftlinge in einer Kleinstadt ableitet, die sich blind und taub stellt, wählt er einen noch polemischeren Begriff, als er die Konfrontation der Amerikaner mit den elenden und ausgehungerten KZÜberlebenden darstellt. Die rein sprachlich bestehende Wirklichkeit erscheint hier in einer Metamorphose, bei der Menschen plötzlich als Tiere erscheinen. Aus der Sicht der satten Sieger sind diese ausgemergelten, zerlumpten und erniedrigten Menschen nur „Blindschleichen“.

Da schüttelt der Amerikaner den Kopf, die Zigarette fällt ihm aus dem Mund, schon sind einige Blindschleichen zur Stelle und balgen sich um den glühenden Stummel. Da nimmt der Amerikaner seine Kamera zur Hilfe, die soll behalten, was das Auge nicht faßt. Er winkt den Schlürfenden, und seine Hand begehrt, dass sie eine Gruppe bilden ${ }^{44}$.

Adler gelingt es in dem Passus, der in seinem Kontext gelesen werden muss, nicht etwa die Menschlichkeit der verhungerten Überlebenden in Frage zu stellen, sondern in dem abschätzigen Blick des Siegers das Unverständnis und den Mangel an Mitgefühl, ja die Verachtung des Siegers zu stigmatisieren, der nur an das außergewöhnliche Foto denkt. Die Blindschleiche ist als Tier ekelhaft, aber wehrlos, da ohne Giftzahn. Ein Gewürm, das man risikolos zertreten könnte. Die „Verwandlung“ der Besiegten in eine Blindschleiche muss in dem Kontext all der extrem kritischen Äußerungen zu den amerikanischen Befreiern gelesen werden, die darin gipfeln, dass Paul zu dem zynischen Oberkommandanten Dudley sagt:

„Entschuldigen Sie, ich habe mich geirrt! Ich habe einen Menschen gesucht. Mein Weg war falsch." Beide Amerikaner grinsen und sagen irgendetwas auf Englisch, aber Paul schlägt die Tür zu und hört nichts mehr ${ }^{45}$.

Die Frage nach der Menschlichkeit stellt sich in den Zeugnissen über die NSVerbrechen im Allgemeinen in Zusammenhang mit den Tätern ${ }^{46}$ oder den erniedrigten Opfern $^{47}$. Adler jedoch fragt nach der Menschlichkeit der Befreier, und seine harsche Blindschleichen-Metapher lieferte schon die Antwort: wer Menschen im Elend auf solche Weise verachtet, hat selber sein Menschsein verraten. 


\section{Schlussbemerkung}

chließend lässt sich sagen, dass Adler in diesem Buch aus dem Jahr 1951, in dem er die Vergangenheit der Shoah künstlerisch umsetzt und verfremdet, doppelt Vergangenheitsbewältigung leisten wollte - auf dem kollektiven Handlungsfeld der ästhetischen Vermittlung, sowie im individual-psychologischen Bereich seiner eigenen Geschichte. Tatsache ist, dass eine späte und zögernde Rezeption des 1962 erschienenen Romans, der erst 1999 wieder aufgelegt wurde, bisher keine nachhaltige Einwirkung auf memorielle Bewusstwerdungsprozesse ermöglichte, wie dies bei dem Band über Theresienstadt der Fall gewesen war. Der bevorstehende 100. Geburtstag von Adler im Jahr 2010 wird, so kann man annehmen, die Rezeptionslage verändern und dem Roman verspätet den wichtigen Platz bereiten, der ihm zukommt - habent sua fata libelli. Elias Canetti schrieb zu der Zeit, als der Roman, der 11 Jahre auf seine Publikation wartete, noch unveröffentlicht war:

Es gibt zweifellos unzählige Menschen, die gerade dieses Buch brauchen, dass sie es noch nicht haben können, ist einer jener ganz unsinnigen Züge unseres modernen Lebens, für die man sich aufs Tiefste schämt ${ }^{48}$

\section{NOTES}

1. Theresienstadt 1941- 1945. Antlitz einer Zwangsgemeinschaft (1948, Erstdruck 1955, 2. verbesserte Auflage 1960) Zsolnay Verlag, Wien 1999.

2. Die verheimlichte Wahrheit. Theresienstädter Dokumente, Tübingen, J.C.B. Mohr (Paul Siebeck) 1958.

3. Der verwaltete Mensch. Studien zur Deportation der Juden aus Deutschland, Tübingen, J.C.B. Mohr (Paul Siebeck), (Niederschrift : 1958-1973), 1974.

4. «Es ist klar, dass man millionenfachen Mord nicht «bewältigen» kann. Die Ohnmacht der Gerichtsverfahren gegen die Täter wegen der Größenordnung ihrer Verbrechen in dieser Vergangenheit beweist diesen Tatbestand in symbolischer Verdichtung.» Alexander und Margarete Mitscherlich, Die Unfähigkeit zu trauern. Grundlagen kollektiven Verhaltens, (1967), München, Piper, 1977, 17. Auflage 2004, S. 24.

5. Peter Reichel: Vergangenheitsbewältigung in Deutschland. Die Auseinandersetzung mit der NSDiktatur von 1945 bis heute, Beck, München, 2001, S. 9.

6. Dieser Begriff beinhaltet keine Gleichstellung des Autors mit dem Freudschen Patienten und seiner Amnesie in der Schrift „Erinnern, Wiederholen Durcharbeiten“. Sigmund Freud, „Erinnern, Wiederholend Durcharbeiten“, in: Schriften zur Behandlungstechnik, Studienausgabe, Ergänzungsband, Frankfurt/Main, Fischer, 1994, S. 205-215.

7. „Warum habe ich mein Buch Theresienstadt 1941-1945 geschrieben?“ in: H. G. Adler: Der Wahrheit verpflichtet, hrsg von Jeremy Adler, Gerlingen Bleicher, 1998, S. 111.

8. S. hierzu Marcel Atze: „Ortlose Botschaft“. Der Freundeskreis H. G. Adler, Elias Canetti und Franz Baermann Steiner im englischen Exil, Marbacher Magazin 84/ 1998, Marbach, Deutsche Schillergesellschaft, 1998, S. 151-153. 
9. Marcel Atze: «'Wie Adler berichtet'. Das Werk H. G. Adlers als Gedächtnisspeicher der Literatur.» Text und Kritik VII/04, 163, H. G. Adler, Göttingen, edition text und kritik, 2004, S. 17. 10. Darunter war auch der Maler Jehuda Bacon, der damals 15 war, und den Adler entscheidend förderte. S. Jehuda Bacon: «Erinnerungen an H. G. Adler», in: Zuhause im Exil, Zu Werk und Person H.G. Adlers, Hrsg. Heinrich Hubmann und Alfred Otto Lanz, Stuttgart, Franz Steiner, 1987, S. 168-169.

11. Zu den Existenzbedingungen der ersten Jahre in London, s. Marcel Atze: «Ortlose Botschaft». Der Freundeskreis H. G. Adler, Elias Canetti und Franz Baermann Steiner im englischen Exil, Marbacher Magazin 84/ 1998, Marbach, Deutsche Schillergesellschaft, 1998, S. 137. Adlers zweite Frau, die Bildhauerin Bettina Gross aus Prag, unterstützte Adler entscheidend auf seinem Weg als Wissenschaftler und Künstler.

12. Joachim Campe: «Der Standpunkt der Verfolgten. Über den Historiker und Erzähler H. G. Adler», in: Zu Hause im Exil. Zu Werk und Person H. G. Adlers, Hrsg. Heinrich Hubmann und Alfred Otto Lanz, Stuttgart, Franz Steiner, 1987, S. 81.

13. Alexander und Margarete Mitscherlich: Die Unfähigkeit zu trauern. Grundlagen kollektiven Verhaltens, (1967), München, Piper, 1977, 17. Auflage 2004.

14. Das Buch erschien nach langen Bemühungen erst 1962 in dem Verlag Bibliotheca Christiana in Bonn und wurde kein großer Verkaufserfolg. 1999 gab der Zsolnay Verlag in Wien eine neue gebundene Ausgabe heraus, inzwischen liegt das Taschenbuch des Aufbauverlages von 2002 vor.

15. Panorama. Roman (1948, Erstdruck 1968), München, Piper, 1988.

16. Zitiert nach Jeremy Adler: „Nachwort. Nur wer die Reise wagt, findet nach Hause“, in: H. G. Adler, Eine Reise, Berlin, Aufbauverlag, 2002, S. 355. Doderer fügt noch an, dass er auch den Untertitel Ballade gerechtfertigt finde, da diese «zum Kristall der Form» schließe. S. Ebd.

17. Iris Denneler: Von Namen und Dingen. Erkundungen zur Rolle des Ich in der Literatur, Königshausen \& Neumann, Würzburg, 2001, S. 7-8.

18. Ebd.,S. 22.

19. „Zerline ist fort. Die Reise hin ist nimmer auszumessen. Die Hand versagt, die jedem einst gezeigt hat. Alle sind sie fort. Zerline ist bei Leopold. Ewiges Ruhenthal ist ihr beschieden im Schatten des Leitenberges." Eine Reise, S. 265.

20. Ebd., S. 27.

21. Eine Reise, S. 34-35.

22. «Der Familienname der beiden Schwestern - Schmerzenreich - beschwört den Erbteil der gesamten Schicksalsgemeinschaft. Ihr Jammertal ist ein Reich des Schmerzens.» J. Adler: Nachwort zu Eine Reise, a.a.0., S. 357.

23. Johann Wolfgang von Goethe: Faust, Erster Teil, Eine Tragödie, Hamburger Ausgabe, Band 3, München, dtv, 1998, S. 114.

24. Hermann Broch: Die Erzählung der Magd Zerline, (1950) Frankfurt, Suhrkamp, 1988. Adler war mit Broch bekannt, wovon der Briefwechsel zeugt. H. G. Adler und Hermann Broch: Zwei Schriftsteller im Exil, Briefwechsel Göttingen, Wallstein, 2004.

25. Siehe auch Jeremy Adler: «Die Macht des Guten im Rachen des Bösen. H. G. Adler: Adorno und die Darstellung der Shoah.» Merkur 6, 54.Jg. 2000, S.481.

26. Eine Reise, S. 14.

27. Jeremy Adler: Nachwort zu Eine Reise, a .a. O., S. 357.

28. «Elle renvoie à un contexte ou situation de l'énoncé, c'est-à-dire non pas tant au monde perçu et imaginé auquel le destinateur et destinataire peuvent se référer qu'au quelqu'un et quelque chose [...] dont on parle et qui n'a d'existence que dans le discours». Jean-Michel Adam, JeanPierre Goldenstein: Linguistique et discours littéraire. Théorie et pratique des textes, Paris, Larousse, 1976, S. 262 .

29. Ebd., S. 44. 
30. Treue, wir sind verläßlich, keinem wurde ein Härchen gekrümmt, jetzt fahrt ihr in die Sicherheit, Heimat, ewige Freiheit, für euer Wohl sorgen, Brüder.

31. «Un énoncé ironique est un énoncé par lequel on dit autre chose que ce que l'on pense ne faisant comprendre autre chose que ce que l'on dit. Il fonctionne comme subversion du discours de l'autre: on emprunte à l'adversaire la littéralité de ses énoncés, mais en introduisant un décalage de contexte, de style ou de ton, qui les rende virtuellement absurdes, odieux ou ridicules, et qui exprime implicitement le désaccord de l'énonciateur». Philippe Lejeune: «Le récit d'enfance ironique : Vallès», in : Je est un autre, Le Seuil, Paris, 1980, S. 24-25. Übersetzung von R.V-K. Siehe auch Florence Leca-Mercier, L'ironie, Hachette, Paris, 2003, S. 66.

32. Eine Reise, S. 33

33. «Nach einer kurzen Weile kommen die verbotenen Menschen zu zweien, gebückt und mit ihrem Gepäck beladen, zum Tor heraus. Sie rufen eine Nummer und ihren ehemaligen Namen. [...] Die Verbotenen versammeln sich auf dem Hof und stellen sich in Viererreihen auf. Zusammen sind es tausend ehemalige Menschen. Der Schielende tritt vor die Reihen, wendet seine Peitsche um und reitet ohne Pferd langsam die Front entlang» Ebd., S. 36.

34. Eine Reise, S.35.

35. Ebd., S. 236.

36. Ebd., S. 339.

37. Eine Ausnahme bildet allerdings die Nennung der Amerikaner als Nation der (skeptisch gesehenen) Befreier.

38. «Die Fremdlinge stehen frech und breitspurig in der Wohnung und weiden sich unbekümmert geschäftig an einem Anblick, den sie doch nicht zu verstehen scheinen.» Eine Reise, S. 12.

39. «Die Fremdlinge haben gedämpfte Taschenlampen, die sie weit von sich weghalten, um das Dunkel auf der Treppe zu durchdringen. Sie tun Böses, ohne diesmal es ausdrücklich zu wollen.» Ebd., S. 23.

40. Die Kranken werden nicht geschont: «Der Schielende las die Befunde dem Helden vor, der befahl, dass niemand liegen bleiben dürfe, der nicht 39 Fieber habe.» Ebd. S. 38. Der Ton des Helden seinem Untergebenen gegenüber spricht für sich : «Da brüllte der Held noch lauter : Maul halten, Schweinehund! ...Nächstes Mal schlag ich Ihnen die Knochen krumm, wenn die Befunde nicht besser geschrieben sind !»Ebd., S. 37

41. «An den fehlenden Worten wird das Unmaß des Grauens gemessen. In diesem ironischen Verfahren wirken nette Worte wie Satire. Die Schergen treten im Roman so auf, wie sie selbst genannt werden wollen. Sie heißen einfach 'Helden'.» Jeremy Adler, Nachwort zu Eine Reise, a.a. O., S. 358.

42. Eine Reise, S. 147.

43. «Einzelne Geister wollen sich nicht dem 'links, rechts' anbequemen, [...], aber die andern Geister verderben ihnen dieses Spiel, weil sie den Leitenbergern wahrhaftig erscheinen wollen, damit wenigstens ein Teil der Städter gestehen muß: Ich habe sie gesehen. Es gibt sie wirklich. Wir haben uns selbst davon überzeugt. Doch die Geister bemühen sich vergeblich. Was sie auch beginnen, sie können es doch nicht erreichen, daß die Leitenberger sie für wirklich halten. [...] Durch die große Anzahl der Geister wird ihr Dasein besonders unglaubwürdig.» Ebd. S. 109.

44. Eine Reise, S. 275.

45. Eine Reise, S. 312. Robert Antelme, dies sei nebenbei angemerkt, beschreibt, wie die Unmöglichkeit der Verständigung über die Erfahrung des univers concentrationnaire Lagerhäftlinge und amerikanische Befreier trennte. Bei Adler wie bei Antelme wird das Englische eingesetzt als Zeichen des «dialogue de sourds» und der monologischen Kommunikationssituation. Robert Antelme, L'espèce humaine, Paris, Gallimard, 1957, S. 301-302.

46. Siehe z.B. Elio Vittorini: Uomini e no, (1945) Milano, Mondadori, 1990.

47. Siehe z.B. Primo Levi: Se questo é un uomo, (1947) Milano, Einaudi, 1981. 
48. Helmut Göbel: «H. G. Adler und Elias Canetti», Text und Kritik VII/04, 163, H. G. Adler, Göttingen, edition text und kritik, 2004, S. 77.

\section{RÉSUMÉS}

H. G. Adler (Prag 1910- London 1988), ein Überlebender der NS- Konzentrationslager, in denen seine Frau und seine ganze Familie ermordet wurden, widmete dem Schreiben über die Shoah einen großen Teil seines Lebens. Seine historischen Bücher über Theresienstadt und seine literarischen Werke, insbesondere der Roman Eine Reise, der 1951 verfasst wurde, bilden ein Panorama des univers concentrationnaire. Die Besonderheit von Eine Reise liegt darin begründet, dass der Roman mit der Darstellung einer fremden und beängstigenden Welt ohne explizite Referenz auf die Realität von der historischen Wirklichkeit abgekoppelt ist. Dem Leser erschließen sich jedoch Verwandtschaften zwischen der «Reise» und der Deportation, den «Verbotenen" und den Juden, den «Fremdlingen » und den SS. In dem Artikel werden die Adlerschen Schreibstrategien untersucht.

H. G. Adler (Prague 1910 - Londres 1988), survivant des camps nazis où ont péri sa femme et toute sa famille, a consacré une grande partie de sa vie à l'écriture de la Shoah. Ses publications historiques sur le camp de Theresienstadt et ses œuvres littéraires, notamment le roman Eine Reise de 1951, constituent un panorama de l'univers concentrationnaire. La particularité de Eine Reise réside dans une déconnexion de la réalité historique à travers la représentation d'un monde étrange et angoissant, sans référent réel explicite. Le lecteur perçoit cependant la similitude entre le voyage («Reise ») et la déportation, entre les «personnes interdites » et les Juifs, entre les « étrangers» et les SS. L'article s'attache à analyser les stratégies d'écriture adlériennes.

INDEX

oeuvrecitee Eine Reise

\section{AUTEURS}

\section{RUTH VOGEL-KLEIN}

ENS Paris 